\title{
La Chine des années 2000 : regards nouveaux sur le politique
}

Sébastien Billioud et Joël Thoraval

\section{(2) OpenEdition}

12 Journals

Édition électronique

URL : http://journals.openedition.org/extremeorient/126

DOI : 10.4000/extremeorient.126

ISSN : 2108-7105

Éditeur

Presses universitaires de Vincennes

Édition imprimée

Date de publication : 1 octobre 2009

Pagination : 5-31

ISBN : 978-2-84292-237-5

ISSN : 0754-5010

Référence électronique

Sébastien Billioud et Joël Thoraval, « La Chine des années 2000 : regards nouveaux sur le politique », Extrême-Orient Extrême-Occident [En ligne], 31 | 2009, mis en ligne le 01 octobre 2012, consulté le 10 décembre 2020. URL : http://journals.openedition.org/extremeorient/126 ; DOI : https://doi.org/ 10.4000/extremeorient. 126 


\title{
La Chine des années 2000 : regards nouveaux sur le politique
}

\author{
Sébastien Billioud et Joël Thoraval
}

En présentant un ensemble de textes récents d'auteurs chinois qu'ils avaient sollicités pour contribuer à une réflexion sur «le politique aujourd'hui en Chine», les deux responsables de ce numéro sont conscients des obstacles que peut rencontrer leur compréhension chez le lecteur français. Ces difficultés tiennent à trois circonstances.

En dépit de la grande diversité de leurs perspectives, la majorité des six auteurs ici réunis ont en commun le désir d'intervenir moins en spécialistes d'une discipline académique qu'en tant qu'intellectuels engagés. S'ils sont par ailleurs également les auteurs d'études répondant aux normes académiques habituelles, ils ont choisi de proposer ici des contributions qui occupent en quelque sorte une position intermédiaire entre la discussion scientifique et le débat politique, en restant sensibles à la multiplicité des mouvements sociaux qui agitent aujourd'hui la société chinoise. Ces textes de combat portent donc nécessairement la marque du style et de la personnalité de leurs auteurs.

Il faut d'autre part prendre en compte les spécificités de l'espace où se trouvent confinés les débats intellectuels dans la Chine actuelle. Son caractère essentiel est probablement ce que l'on pourrait nommer aussi bien «l'omniabsence» que l'omniprésence de l'appareil idéologique communiste. Cette circonstance confère parfois une certaine irréalité au débat puisque, si le Parti n'y est presque jamais présent «en personne», par le truchement d'un idéologue clairement assigné à cette fin, il ne cesse de le surdéterminer en permanence, ne serait-ce que de manière négative. En conséquence, à l'exception de la contribution de l'intellectuel taïwanais Lee Ming-huei, qui jouit des conditions d'une société démocratique, les textes ici présentés sont souvent remarquables par leurs omissions ou par leur caractère allusif. On verra que le langage crypté de certains peut être le simple effet de la nécessité ou obéir au contraire à des stratégies délibérées. 
Mais il est une difficulté supplémentaire dont il faut également tenir compte. Il est en effet remarquable, et c'est sans doute une des caractéristiques de ces années 2000, que la plupart de ces intellectuels éprouvent le besoin de légitimer leurs positions théoriques par des références croissantes à la longue tradition culturelle et intellectuelle de la Chine, qu'il s'agisse de s'en réclamer ou de prendre ses distances avec elle. Le lecteur français voit donc sa lecture rendue parfois malaisée par de multiples références faites à l'histoire ou à la pensée de la Chine impériale, alors même que les enjeux restent contemporains.

En conséquence, il ne s'agira pas ici de proposer un tableau synthétique sur l'état de la réflexion politique aujourd'hui en Chine. Cela ne serait du reste pas possible, compte tenu des choix opérés dans la sélection des articles, sur lesquels la conclusion de ce texte apportera des précisions nécessaires. On se consacrera donc à une tâche plus modeste: rendre le plus lisibles possible les contributions des intellectuels chinois présentées dans ce recueil, en retraçant les contextes, en explicitant allusions et références et en offrant, de manière au moins provisoire, quelques réflexions sur les multiples dimensions de cette pensée chinoise du politique au cours de la présente décennie.

\section{Quand la nécessité d'une relecture du confucianisme s'impose dans le débat intellectuel}

Dans une large mesure, et quoiqu'il soit à maints égards nécessaire d'en réévaluer l'histoire, le confucianisme apparaît comme la grande victime des entreprises de modernisation de la Chine du $\mathrm{XX}^{\mathrm{e}}$ siècle. Dénoncé tant par les héritiers libéraux du mouvement du 4 mai 1919 que par ses héritiers communistes, il a été congédié d'une histoire tournant le dos au passé et dominée par de grands récits émancipateurs inspirés par l'Occident. Il s'est progressivement fragmenté en projets religieux (avec la tentative d'institutionnaliser une religion confucéenne et le développement d'organisations populaires syncrétistes), idéologiques (au travers de mobilisations entreprises par le Guomindang comme le «Mouvement de la vie nouvelle» de 1934 ou celui, lancé en 1966 à Taiwan, de la renaissance de la culture chinoise) mais aussi intellectuels : dans ce dernier domaine, il a pris la forme d'entreprises philosophiques majeures quoique souvent marginalisées au sein du monde académique. L'ouverture de la Chine a profondément changé la situation et les années 2000 voient le retour en force, déjà amorcé pendant la décennie précédente, du confucianisme dans les courants de pensée les plus variés du monde intellectuel.

C'est à la périphérie du monde chinois, principalement à Taiwan et Hong Kong, que le confucianisme s'est affirmé comme projet philosophique à travers 
le mouvement dit du «néo-confucianisme contemporain ${ }^{1}{ }$. Ce mouvement rassemble des individus très différents qui partagent néanmoins la conviction que la culture chinoise - et au premier chef le confucianisme - demeurent des ressources spirituelles vivantes, susceptibles de contribuer à la modernisation chinoise. En 1958, quatre de ses éminents représentants, Tang Junyi, Mou Zongsan, Xu Fuguan et Zhang Junmai rédigent un manifeste destiné autant à mobiliser les intellectuels chinois qu'à critiquer les interprétations des sinologues occidentaux sur le caractère historiquement dépassé de la vision confucéenne, condamnée selon l'historien Joseph Levenson à une inévitable «muséification». L'un des traits principaux de ce mouvement, dont Lee Ming-huei dans le présent volume est d'une certaine manière le représentant, est de s'inscrire dans l'héritage des «Lumières chinoises », c'est-à-dire du mouvement du 4 mai 1919 qui en appelait à la science et à la démocratie. En d'autres termes, le néo-confucianisme contemporain n'est pas un mouvement conservateur, perdu dans la nostalgie d'un ordre autocratique révolu. Bien plutôt, ses représentants partagent avec les libéraux quelques conceptions fondamentales, même s'ils récusent chez ces derniers ce qu'ils perçoivent comme une attitude iconoclaste vis-à-vis de la tradition.

Leur réflexion sur la démocratie est à cet égard exemplaire. Mou Zongsan analyse celle-ci comme un universel (un «dharma commun»), ayant donc vocation à se développer en Chine comme ailleurs. Cette reconnaissance s'accompagne de l'acceptation d'une rupture fondamentale au sein de l'ancien idéal que décrivait le classique de l'Antiquité, La Grande Étude. Il n'est plus concevable que le bon ordre sociopolitique puisse être la simple conséquence d'une culture et d'une «extension» des vertus morales: il n'y a désormais plus de continuité possible entre la «sagesse intérieure » (neisheng) à laquelle s'exercent l'individu et son entourage et la «royauté extérieure» (waiwang), c'est-à-dire l'ordre collectif légitime. C'est cette discontinuité qui fait la modernité de ce confucianisme nouveau. Elle est pensée par Mou Zongsan, dans un vocabulaire encore hégélien, sur le mode d'une «auto-négation» de la conscience morale (ziwo kanxian): le passage, chez le sujet, de l'univers éthique et métaphysique à l'univers de la science et de la politique signifie l'entrée dans un type de rationalité nouvelle, de nature instrumentale. Sur le plan collectif, l'éthique confucéenne ne peut plus désormais légitimer une vision pan-moraliste du politique, placée

1. Voir Joël Thoraval, «La Chine et le confucianisme au défi de la modernité», Esprit, 265, juillet 2000, p. 140-154. John Makeham, New Confucianism, New York, Palgrave Macmillan, 2003. Umberto Bresciani, Reinventing Confucianism, The New Confucian Movement, Taipei, Taipei Ricci Institute, 2001. Lee Ming-huei, Der Konfuzianismus im modernen China, Leipzig, Leipzig Universitätsverlag, 2001. 
sous l'idéal du Roi-Sage, dont l'histoire de la Chine a montré les méfaits. Si l'éthique confucéenne peut néanmoins prétendre constituer le fondement moral de la démocratie, elle n'exerce plus ses effets que de manière indirecte, au sein d'un univers politique dont elle reconnaît l'autonomie.

La contribution de Lee Ming-huei dans ce numéro permet d'abord, à travers une rétrospective historique prenant en compte les débats commencés dans les années 1950 entre libéraux et confucéens, de prendre la mesure de ce qui rapproche les deux camps avec, au premier chef, leur hostilité profonde à tout projet totalitaire ou autoritaire. Parallèlement, elle dessine l'espace d'un devenir possible du confucianisme, qui trouve particulièrement à s'exercer dans la société démocratisée de Taiwan. Si l'on met à part les formes particulières que le confucianisme populaire revêt sur l'île à travers son appropriation par des mouvements religieux syncrétistes, force est de constater qu'il s'est surtout transformé en entreprise intellectuelle dépourvue d'objectifs sociaux et politiques directs $^{2}$. Ainsi, Lee Ming-huei compare-t-il le rôle joué dans l'arène politique taiwanaise par le néo-confucianisme contemporain à celui d'un John Rawls dans la vie politique américaine. Son œuvre porte elle-même la marque de cette attitude délibérément modeste. Élève de Mou Zongsan, détenteur d'un doctorat soutenu à Bonn sur le «sentiment moral» chez Kant, Lee Ming-huei est un auteur prolifique qui est aujourd'hui, à Taiwan, l'un des principaux représentants du néo-confucianisme contemporain. Mais sa promotion d'un confucianisme démocratique et libéral est avant tout intellectuelle et académique, dépourvue de visée directement politique ou de volonté de transformer ce courant de pensée en mouvement social. À cet égard, on verra que cette situation est bien différente de celle qui prévaut aujourd'hui en Chine continentale.

La politique d'ouverture du pays lancée par Deng Xiaoping à la fin des années 1970 permet un retour de la pensée libérale et l'arrivée nouvelle d'idées et de théories venues de l'Occident. L'attrait du libéralisme est réel pour un monde intellectuel ayant vécu les épisodes traumatiques du maoïsme, et plusieurs des contributeurs de ce numéro, comme Qin Hui, Gan Yang ou Wang Hui se reconnaissent alors dans ce mouvement. Dans la «fièvre culturelle» de l'époque, la «tradition» est parfois redécouverte avec intérêt ${ }^{3}$, mais elle reste le plus souvent associée à une culture «féodale» et autocratique dont on croit

2. Cette affirmation peut néanmoins être nuancée, car il existe un mouvement partant de l'université et visant à promouvoir les textes classiques confucéens dans la population et en particulier chez les enfants.

3. L'Académie de la culture chinoise ouverte en marge de l'Université de Pékin par des spécialistes du confucianisme dans les années 1980 donne l'exemple d'une entreprise de promotion de la culture traditionnelle (initiation aux classiques par exemple) qui connaît un succès certain, mais dans un cercle encore très limité. 
reconnaître la perpétuation dans le régime maoïste. La conscience historique de l'époque reste dominée par une vision téléologique de l'histoire où les promesses du marché se substituent à celles de la société communiste. La nouveauté réside dans la volonté désormais affirmée - et maintenue jusqu'à aujourd'hui par les libéraux - de ménager à l'individu une sphère d'autonomie et de droits face à la toute puissance du pouvoir.

Les événements de Tian'anmen marquent une rupture et ont un impact profond sur le monde intellectuel. D'une part, celui-ci tend à se recentrer sur l'étude, le хиеshu. C'est dans les années 1990 qu'on redécouvre de manière positive le néo-confucianisme contemporain et, plus largement, la «tradition culturelle», dans un climat nouveau de «fièvre des études nationales » (guoxиe re). D'autre part, à partir de 1993, la Chine entre dans une nouvelle phase de son développement économique avec la reprise rapide de la croissance et des flux d'investissements étrangers. Des fractures se creusent au sein de la société chinoise entre la classe moyenne émergente et les laissés pour compte des restructurations. Sur le plan intellectuel, le capitalisme et la mondialisation économique font désormais l'objet d'une critique systématique et approfondie de la part d'une «nouvelle gauche» chinoise (xin zuopai), largement issue du libéralisme des années 1980, mais pour laquelle l'impératif de justice sociale doit primer sur toute autre priorité, notamment sur la réforme institutionnelle et juridique. Sur le plan politique, le gouvernement de Hu Jintao prend acte des risques sociaux courus par le pays en lançant le projet, aux accents vaguement confucéens, d'une «société d'harmonie». De son côté, le camp libéral, quoique affaibli, renouvelle sa réflexion, comme en témoigne l'exemple de Qin Hui, qui reste un «intellectuel public» et défend un modèle chinois à la fois libéral en matière de libertés publiques et social-démocrate dans son attention aux problèmes sociaux.

Né en 1953 et professeur d'histoire à l'Université Tsinghua de Pékin, Qin Hui a été envoyé plusieurs années à la campagne pendant la Révolution culturelle, dans un village du Guangxi. Son combat intellectuel porte profondément la trace de cette expérience vécue. Il présente dans ce recueil une réflexion historique sur le confucianisme dans le but d'en tirer des enseignements pour le présent. Son argumentation est centrée sur la dénonciation d'un mécanisme à l'œuvre dans l'histoire chinoise. Selon lui, l'idéal confucéen du bon gouvernement a été en permanence dévoyé sous la conjonction de deux forces opérant en concurrence, et auxquelles il choisit de donner le nom d'écoles philosophiques rivales apparues dans l'Antiquité, au risque de susciter certaines confusions. En effet, ce qu'il appelle le «légisme» renvoie moins à l'antique école désignée sous ce nom qu'à une attitude politique séculaire visant à soumettre l'individu au contrôle omniprésent d'un pouvoir centralisé. Quant au «taoïsme», il désigne moins une 
pensée singulière qu'une situation dans laquelle des groupes d'intérêts rivaux cherchent à obtenir du pouvoir une politique de plus grand «laisser-faire» leur permettant de tirer avantages et profits. C'est à la lumière de ce modèle assez simple, qu'il appelle le dispositif pervers « légisto-taoïste», que Qin Hui entend analyser des pans entiers de l'histoire chinoise.

Mais ce qu'il faut souligner, c'est la portée actuelle de l'argumentation de Qin Hui, qui s'adresse simultanément à trois interlocuteurs. À la nouvelle gauche qui lutte pour plus de justice, Qin Hui réplique que sans démocratie (sans un rejet de la politique d'inspiration légiste qui se perpétue encore), seuls les groupes d'intérêts (les «taoïstes ») profitent de la libéralisation économique en accaparant les richesses du pays (par exemple les actifs privatisés des entreprises d'État). À ses amis libéraux qui dénoncent aujourd'hui le confucianisme, Qin Hui répond qu'il ne faut plus se tromper de combat, comme le firent trop souvent les intellectuels des «Lumières chinoises» des années 1920 ou ceux des «nouvelles Lumières» des années $1980^{4}$. Ce qu'il faut reconnaître comme adversaire, c'est la «grande communauté» centralisée qui écrase l'individu, non le confucianisme lui-même. En effet, ce qu'on présente souvent sous le nom de confucianisme n'est qu'une idéologie impériale, ayant détourné dans un sens despotique les idéaux originels de ce courant de pensée. Il existe un parallèle, à cet égard, entre l'histoire passée et la situation présente où le symbolisme traditionnel et «confucéen » utilisé par le pouvoir communiste ne sert que de justification à une politique autoritaire. Enfin, aux partisans du confucianisme, Qin Hui adresse également une critique nuancée: les «nouveaux confucéens contemporains » d'inspiration démocratique, évoqués précédemment, ne pourront guère jouer de rôle dans les transformations de la société chinoise s'ils restent étroitement confinés dans leur domaine académique. Quant aux tenants du «confucianisme politique», dont il va être question, Qin Hui les met aussi en garde: le rapprochement avec les valeurs de la démocratie occidentale est le seul moyen pour le confucianisme d'échapper enfin au piège dans lequel le dispositif «légisto-taoïste» l'a longtemps enfermé.

La réflexion critique sur la «tradition» à laquelle se livre Qin Hui doit être comprise, au-delà des discours gouvernementaux ou de l'engouement académique des années 1990 pour les «études nationales», dans un contexte plus général de regain d'intérêt pour le confucianisme en Chine continentale. Ce phénomène nouveau est perceptible à la fois dans la société et dans le monde intellectuel. Dans la société, la référence au confucianisme prend une pluralité

4. On se souvient que les libéraux des années 1980 (dont les «nouvelles Lumières» se veulent explicitement la reprise de celles des années 1920) tendaient à associer les errements du maoïsme à la «tradition féodale». 
de formes qui vont de mouvements d'apprentissage de textes classiques à la réouverture d'institutions éducatives traditionnelles, de la redécouverte de pratiques rituelles à la formation d'associations privées prônant la culture de soi. Dans le monde intellectuel, un courant conservateur milite désormais pour la redécouverte de la dimension politique et religieuse du confucianisme: on évoque la possibilité de sa transformation en religion ou enseignement d'État (guojiao) et certains prônent à demi-mot la transformation du parti communiste en parti confucéen. Si personne ne se fait ouvertement le porte-parole de ces idées au sommet de l'appareil d'État, de telles conceptions se diffusent pourtant au sein du dernier. Qu'il suffise, à titre d'exemple récent, de mentionner le développement rapide d'un enseignement consacré au confucianisme et à la culture traditionnelle à l'école du Parti ainsi que l'engouement exceptionnel qu'il suscite ${ }^{5}$. Des sujets auparavant tabous (comme la transformation du Parti) sont directement abordés et font même l'objet de discussions sur le site Internet de l'institution ${ }^{6}$.

Pour comprendre la spécificité des nouvelles théories politiques inspirées par le confucianisme, il est bon de revenir aux nouveaux confucéens contemporains évoqués un peu plus haut. Soucieux de réaffirmer la valeur d'une tradition spirituelle longtemps mise à mal, ces derniers se sont avant tout employés à reconstruire de savants systèmes de «philosophie première» dans un double rapport d'opposition et de mimétisme avec la philosophie occidentale. Ainsi, dans une magistrale tentative, Mou Zongsan s'efforce de réarticuler le sens de l'ancien idéal de sagesse en recourant à des philosophèmes empruntés au kantisme, comme celui d'intuition intellectuelle. Cette entreprise spéculative met à profit le néo-confucianisme des dynasties Song et Ming, lui-même une réaction au développement en Chine du bouddhisme au cours du premier millénaire de notre ère. Pour les tenants actuels d'un confucianisme politique, la dimension métaphysique de ce courant de pensée n'est guère susceptible de fournir les ressources qui permettraient de poser la question du politique, aujourd'hui en Chine. Il faut pour ce faire recourir à une autre école apparue sous la dynastie des Han : le Gongyang.

La tradition dite du Gongyang est à l'origine une école exégétique ayant établi le commentaire d'un des Classiques de l'Antiquité, les Annales des printemps et automnes. Cette tradition considère que ce texte, loin d'être la simple chronique historique du pays de Lu, dans l'actuel Shandong, est en réalité porteur des enseignements ésotériques de Confucius énonçant en particulier

5. Observation de terrain, Pékin, mars 2009.

6. Sébastien Billioud, "Confucianisme", "tradition culturelle" et discours officiels dans la Chine des années 2000 », Perspectives chinoises n²007/3, p. 50-65. 
des principes pour la fondation d'une dynastie nouvelle. Sous la dynastie des Han, le Gongyang est notamment défendu par Dong Zhongshu (-179, -104 av. J.-C.), un lettré qui joue un rôle clé dans la promotion du confucianisme comme idéologie impériale. Cette tradition connaît ensuite une longue éclipse pour réapparaître à la fin de l'Empire. Ainsi, Kang Youwei (1858-1927) voit dans ce commentaire - et dans l'image d'un Confucius aux accents prophétiques - un moyen de promouvoir son programme de réformes, qui ne pourra qu'être brièvement mis en œuvre en 1898 avec l'assentiment du jeune Empereur Guangxu ${ }^{7}$. Aujourd'hui, le Gongyang continue d'exercer une certaine fascination. Gan Yang, devenu l'un des hérauts de la nouvelle gauche, n'hésite pas à y recourir, comme on le verra, pour donner forme à sa réflexion sur l'historicité complexe de la culture politique chinoise. Liu Xiaofeng, un autre contributeur de ce recueil, lui consacre également de longs développements dans son ouvrage Enseignement confucéen et État-Nation. Mais le plus ardent promoteur du Gongyang est sans aucun doute aujourd'hui Jiang Qing.

Né en 1953, longtemps professeur de philosophie à l'Institut d'administration de Shenzhen, Jiang Qing est aujourd'hui retiré du monde universitaire. Il a créé une académie traditionnelle dans les montagnes du Guizhou dans laquelle il passe une partie de son temps à écrire et enseigner à des disciples. Intellectuel engagé, il contribue également à promouvoir l'étude des textes classiques dans la société, notamment chez les enfants, tout en présentant régulièrement ses idées dans les médias sans toujours néanmoins les exprimer dans toute leur radicalité. Il s'est d'abord fait connaître par une monographie sur l'école du Gongyang, avant de faire paraître à Taiwan des textes alors impubliables en Chine continentale. Un ouvrage de synthèse intitulé Le Confucianisme politique paraît néanmoins en 2003 sur le continent, où il rencontre un large écho. On trouvera, dans le présent recueil, un texte de combat de cet auteur, dans lequel l'adversaire désigné n'est autre que la démocratie occidentale.

Selon Jiang Qing, la question du politique concerne au premier chef le problème de la légitimité. La volonté du peuple ne saurait en être l'unique fondement: il faut que soient pris en compte une certaine transcendance («le Ciel»), ainsi que l'histoire et la culture («la Terre»). L'originalité de Jiang Qing, une fois ces principes arrêtés, est de proposer des mesures institutionnelles concrètes afin de les mettre en œuvre : il avance ainsi l'idée d'un parlement tricaméral au sein duquel chaque chambre représenterait un des types de légitimité et suggère des modes de sélection nouveaux du personnel politique. Il va de soi qu'un tel

7. Sur l'ancienne école du Gongyang et sur sa reprise moderne, voir Anne Cheng, Étude sur le confucianisme Han, Collège de France, Institut des Hautes Études chinoises, 1985, et Histoire de la pensée chinoise, Seuil, 1997, p. 575-583. 
projet ne peut manquer de rencontrer en Chine de très vives oppositions. Cellesci sont manifestes tant chez les libéraux que chez ceux qui restent attachés à certains idéaux du communisme. De fait, la fièvre confucéenne actuelle génère aussi son contraire: un anti-confucianisme qui peut être virulent. Mais il faut aussi souligner que l'opposition aux idées de Jiang Qing se rencontre également chez les confucéens d'inspiration libérale et démocrate, comme en témoigne la fin de l'article de Lee Ming-huei dans le présent recueil.

Le retour du confucianisme dans le débat intellectuel prend parfois des formes inattendues, comme le montre l'itinéraire d'un Gan Yang, devenu aujourd'hui l'un des représentants de la nouvelle gauche. Né en 1952, Gan Yang est séduit par le libéralisme dans les années 1980, comme beaucoup d'intellectuels de sa génération. Il est alors le directeur d'un des périodiques les plus influents de cette décennie: Culture: la Chine et le monde (Wenhua: Zhongguo yu shijie). À cette époque, il réfléchit notamment au rôle de la tradition, refusant de l'essentialiser et proposant de la considérer dans sa dynamique et sa transformation, ainsi que dans son orientation vers l'avenir ${ }^{8}$. Son attaque contre le confucianisme est alors frontale: "Après le $\mathrm{xx}^{\mathrm{e}}$ siècle, demande-t-il, la "tradition" culturelle chinoise pourra-t-elle encore être symbolisée et représentée par le confucianisme ? Nous récusons sans appel cette possibilité ${ }^{9}$.» Il infléchit certes cette position dans les lignes qui suivent, en expliquant que le confucianisme sera toujours une composante parmi beaucoup d'autres de la tradition chinoise. Mais, dans tous les cas, il en exclut toute renaissance ultérieure ${ }^{10}$. On ne manquera dès lors pas d'être surpris de découvrir dans le texte publié ici l'apologie d'une République socialiste confucéenne... Si la conception du confucianisme évoquée dans le texte demeure à un niveau de généralité qui offre peu de prise au commentaire (sont par exemple évoquées la «valorisation des relations interpersonnelles», les «traditions locales» ou encore familiales), la simple présence de cette référence dans le slogan proposé porte sans doute la marque d'un changement caractéristique de l'époque nouvelle: la réémergence du confucianisme s'accompagne en effet d'un remaniement d'ensemble de la mémoire historique.

8. Gan Yang, «Chuantong, shijianxing yu weilai» (Tradition, temporalité et avenir), in Dushu, 1986/2. Consultable sur: http://www.tecn.cn/data/detail.php?id=9103 (lien consulté le 18 juin 2009).

9. Ibid.

10. Ibid. 


\section{Perceptions nouvelles de l'historicité}

Ces perspectives nouvelles sur les rapports entre tradition culturelle et démocratie sont contemporaines d'une perception renouvelée de l'historicité. Tout se passe comme si, à partir des années 1990, il avait fallu se libérer d'une conception moniste et déterministe de l'histoire héritée de la période maoïste et qui pesait encore d'un poids très lourd sur le mouvement démocratique des années 1980. De fait, les événements de la Place Tian'anmen ont été vécus par certains jeunes intellectuels comme l'expression d'un volontarisme héroïque seul à même de mettre en échec un fatalisme historique, celui d'une accablante tradition «féodale» perpétuée par le despotisme communiste.

Ce que rendent possible les années 1990, c'est une remise en cause des «grands récits » en appelant aux « lois de l'histoire», dans un climat désormais influencé par les diverses moutures de la pensée post-moderne. Certes, l'horizon du moderne et le projet des Lumières restent vivants chez certains intellectuels comme Qin Hui : pour lui comme pour Habermas, la modernité reste un «projet inachevé» et l'idée d'une nécessaire modernisation politique de la Chine est plus que jamais à l'ordre du jour. Mais cette perspective émancipatrice doit désormais prendre en compte les critiques du projet moderne énoncées, par exemple, au nom de la pensée post-coloniale ou de la remise en cause de «l'orientalisme» (Edward Saïd étant un des auteurs les plus populaires de la décennie 90).

On choisira ici de relever, parmi les textes proposés, trois aspects de cette perception nouvelle de l'historicité dans les années 2000 : une pensée n'hésitant pas à trouver ses ressources dans le «pré-moderne» (Jiang Qing), une posture se réclamant de manière rigoureuse de l' «anti-moderne» (Liu Xiaofeng) et une volonté de réconcilier les mémoires rivales de l'histoire chinoise au profit d'une articulation renouvelée de «trois traditions » (Gan Yang).

La revendication par Jiang Qing d'un «confucianisme politique», au nom de la tradition exégétique du Gongyang, se situe elle-même dans le contexte plus vaste de reconstructions imaginaires de la tradition pouvant donner lieu à divers mouvements utopiques. C'est à un véritable saut hors de l'historicité que l'on assiste parfois, comme dans le cas d'un intellectuel, non représenté dans ce recueil, comme Zhang Xianglong, professeur de philosophie à l'Université de Pékin. Cet universitaire est, en effet, progressivement passé d'une critique des limites de l'institution académique moderne (où les départements de philosophie ne permettent pas de faire droit à l'idéal d'auto-transformation proposé par la pensée néo-confucéenne) à la revendication d'une véritable communauté territoriale que l'État laisserait s'auto-organiser selon des principes traditionnels, voire archaïques (lignage, ritualisme, technologie préindustrielle, etc.), sur le modèle des villages Amish de Pennsylvanie. C'est un univers micro-politique 
qui existerait ainsi en marge de la société globale, en rupture avec les idéaux de développement et d'administration technocratique qui inspirent cette dernière... Si ces perspectives visionnaires, développées et défendues avec cohérence et obstination, ne peuvent que soulever le scepticisme, elles témoignent aussi d'une sorte d'élargissement des possibles, d'une forme d'imaginaire politique qui aurait été impensable une décennie auparavant.

Le projet de Jiang Qing, que l'on a introduit plus haut, présente des aspects plus réalistes. Mais il n'est pas loin, en raison de sa référence à la vision transmise par le Gongyang, de se placer dans une perspective méta-historique. À vrai dire, une telle pensée fonctionne simultanément à deux niveaux. Il existe d'abord des vérités ou des valeurs éternelles: «nos anciens sages ont fixé de manière définitive les principes du politique». Ces valeurs sont les normes suprêmes, représentées par l'idée de zhengdao, définissant la possibilité d'une communauté politique légitime, au triple point de vue développé par l'auteur (sacré, historique et populaire). Mais ce dogmatisme de principe s'accompagne d'une exigence de créativité et d'expérimentation en ce qui concerne la vie politique elle-même, le zhidao, c'est-à-dire les institutions et les pratiques politiques. De fait, le discours de Jiang Qing associe en permanence deux niveaux d'intelligibilité: ainsi, une critique très empirique des travers du suffrage universel (selon un argumentaire sans surprise) s'accompagne d'une référence sous-jacente à des oppositions normatives ( «désirs humains » contre «voie du Ciel») qui sont de nature métaphysique et, en dernière analyse, religieuse.

Il existe donc, à côté de positions «méta-historiques» (inspirées par une eschatologie héritée de la tradition du Gongyang), tout un versant pragmatique et réaliste de cette pensée, invitant à des discussions institutionnelles et juridiques où la formation de juriste de Jiang Qing trouve à s'exercer. C'est un aspect un peu inattendu de son entreprise que de pouvoir ainsi, en dépit de développements abrupts et d'une présentation caricaturale de la démocratie, donner lieu en Chine à des discussions qui prennent en compte les problèmes concrets de la société et rencontrent d'autre part un écho, notamment nord-américain, qui ne peut laisser indifférent ${ }^{11}$.

À distance d'une telle entreprise (mais non sans certaines affinités), un discours se définissant comme explicitement anti-moderne a été élaboré, avec une grande rigueur, par un intellectuel comme Liu Xiaofeng. Les motivations

11. Voir Daniel A. Bell, China's New Confucianism. Politics and Everyday Life in a Changing Society, Princeton, Princeton University Press, 2008 et Fan Ruiping (éd.), Rujia shehui yu daotong fuxing; yu Jiang Qing duihua (Société confucéenne et renaissance de la transmission du Dao; dialogues avec Jiang Qing), Shanghai, Huadong shifan daxue chubanshe, 2009. 
du texte présenté dans ce numéro sur «Leo Strauss et la Chine» restent cependant implicites et demandent à être éclairées par le parcours de l'auteur et l'évolution de ses idées. Né en 1956, Liu Xiaofeng a d'abord été formé, à l'Université de Pékin, à ce qui s'appelait alors la «poétique comparée». La lecture de Dostoïevski puis des philosophes allemands le guérit, dit-il, du «problème chinois » : dans la rencontre entre Chine et Occident, ce qui compte, c'est l'esprit absolu, non l'esprit national ${ }^{12}$. Des études en théologie et philosophie religieuse à Bâle confortent son aversion pour le relativisme culturel et font de lui un adepte de ce qu' on nomme dans les années 1990 le «christianisme culturel» (wenhua jidujiao), c'est-à-dire une attirance d'ordre intellectuel pour les valeurs du christianisme qui ne se traduit pas nécessairement par une pratique religieuse. Il s'illustre en particulier dans une polémique contre la «théologie indigénisée» (bense shenxue), développée notamment à l' «Institute of Sinochristian Studies » de Hong-Kong, pour y défendre une «théologie en langue chinoise » (hanyu shenxue) qui refuse la sinisation du message chrétien. En effet, l'idée d'une théologie sinisée repose sur le préjugé selon lequel le christianisme est le produit de la culture occidentale. Or, l'événement-Christ (pensé avec la radicalité d'un Karl Barth) est une interruption de l'histoire humaine qui met en question toute culture historique, qu'elle soit juive, grecque, latine... ou chinoise ${ }^{13}$. Mais c'est la rencontre avec la pensée de Leo Strauss qui va donner une consistance théorique à la radicalité anti-culturaliste de Liu Xiaofeng. Aux sources de la culture occidentale se trouvent deux absolus, exprimés à Athènes et à Jérusalem: la raison philosophique et la révélation religieuse ${ }^{14}$. C'est en les comprenant dans leurs propres termes, par delà tous les commentaires modernes, qu'il faut à la fois connaître et vivre la tension irréductible de ces deux types de vérités éternelles.

Le texte présenté ici consiste en une attaque frontale à la fois contre la modernité intellectuelle et politique et contre l'institution académique qui en est le soutien. Le retour aux vérités antiques implique la mise en question systématique du relativisme et de l'historicisme des Modernes. Ce sont les Lumières qui sont ici contestées ainsi que ce qui constitue, aux yeux de l'auteur, leurs

12. Voir le fragment autobiographique, en préface à l'édition revue de Zhengjiu yи хіаoуao (Delivering and Dallying), Shanghai, Shanghai sanlian shudian, 2001, p. 1-10.

13. Liu Xiaofeng, préface à Dao yu Yan, Huaxia wenhua yu jidu wenhua xianyü (La Voie et le Verbe, La rencontre entre la culture chinoise et la culture chrétienne), Sanlian shudian, verbe, p. 1-9.

14. Il est symptomatique de la fascination pour la pensée de Strauss qu'une revue philosophique fondée dans les années 2000 à l'Université de Hainan par d'anciens chefs des Gardes rouges, comme le philosophe Zhang Zhiyang, ait choisi de s'intituler Qishi yu lixing (Révélation et raison). 
prolongements philosophiques et scientifiques: marxisme, sciences humaines, post-modernisme. Dans ce texte, la réaction anti-moderne de Liu prend la forme d'une mise en cause systématique de l'attitude anthropologique qui, avec des hellénistes comme Jean-Pierre Vernant, porte le relativisme culturel et historique au cœur même des humanités classiques. Au lieu d'être abordée dans son contenu de vérité, la doctrine des anciens sages est traitée dans une perspective objectivante et axiologiquement neutre. L'institution universitaire moderne est imprégnée de cet esprit relativiste, qui porte en germe le nihilisme, et rend la jeunesse instruite incapable de tout jugement en valeur. Si la pensée de Leo Strauss est particulièrement nécessaire en Chine, c'est que l'institution académique chinoise n'est pas encore parvenue à s'établir pleinement selon les normes du savoir standardisé de l'université moderne : cette faiblesse est paradoxalement une force, qui rend d'autant plus souhaitable un accès direct aux vérités des Anciens et une critique des diverses herméneutiques modernes.

Pourquoi cette posture anti-moderne se réclame-t-elle d'une "philosophie politique» d'inspiration straussienne? Parce qu'avec la mise à mort de Socrate, la philosophie découvre qu'elle doit se protéger contre la Cité qui lui a donné naissance. Le tournant politique qu' amorce Platon renvoie moins à une doctrine politique particulière au sein du système philosophique qu'à une décision d'autoprotection du philosophe. Socrate a eu tort de croire que la critique radicale des valeurs de l'homme ordinaire pouvait être abordée de manière ouverte sur l'agora. La vie philosophique, selon les vérités éternelles des anciens sages, ne peut être que le fait d'une minorité, qui ne peut suivre ces exigences ultimes que protégée contre le monde menaçant de l'opinion commune. Ce «tournant politique» de la philosophie est le résultat d'une prise de conscience que notre intellectuel chinois reprend à son compte: le danger qui menace la vie selon la sagesse ne vient pas seulement d'en haut, des autorités politiques et idéologiques, mais aussi d'en bas, du peuple lui-même ${ }^{15}$. En conséquence, l'attitude straussienne de Liu Xiaofeng n'est pas seulement anti-moderne, elle est aussi foncièrement anti-démocratique.

15. Voir notamment Liu Xiaofeng, «Ciwei de wenshun, Bolin he Shitelaosi» (La docilité du hérisson: Berlin et Strauss), in Qishi yu lixing (Révelation et raison), dossier «Cong Sukelati, Nicai dao Shitelaosi» (De Socrate et Nietzsche à Strauss), Zhongguo shekui kexueyuan, 2001, p. 1-64. Outre de nombreux textes de Strauss, ont été traduits en chinois dans les années 2000 les travaux de Heinrich Meier relatifs à Carl Schmitt et à Leo Strauss: Maier Heinrich, «What is political theology?», in Leo Strauss and the Theologico-Political Problem, Cambridge (Royaume-Uni), Cambridge University Press, 2006, p. 75-87; Meier Heinrich, Carl Schmitt, Leo Strauss et la notion de politique, Un dialogue entre absents, Paris, Julliard, 1990. 
Si cette attitude ne se laisse lire qu'en filigrane, c'est précisément en raison de cette nécessité d'auto-protection. En bon lecteur de Persecution and the Art of Writing, des intellectuels comme Liu ont fait leur la nécessité d'un double discours, exotérique et ésotérique, selon qu'il s'adresse à une élite ou à un large public. L'intention sous-jacente est de favoriser la naissance d'une aristocratie intellectuelle au sein même de la société de masse, dans un esprit clairement indiqué par Leo Strauss ${ }^{16}$. La critique radicale des institutions universitaires doit être comprise dans cette perspective. Quant au caractère tranchant donné au rejet des théories modernes et post-modernes dans cet article, il est dû au fait que la poursuite de la vérité des anciens résulte moins d'un argumentaire que d'un «choix» : c'est une décision, qui dépend du caractère, car tout le monde n'est pas apte à la «vie philosophique». La lecture de Carl Schmitt ne peut que conforter chez Liu Xiaofeng la dimension «décisionniste» d'une telle orientation intellectuelle.

De là le caractère plus ou moins explicite ou plus ou moins réservé des textes publiés par cet auteur. À usage interne peuvent être discutés, entre pairs, des problèmes relevant d'une théologie politique fortement influencée par Schmitt ; une prudence est cependant de rigueur, en raison du contrôle non seulement des autorités idéologiques mais aussi des réactions publiques, s'inquiétant par exemple sur Internet, à partir de 2005, des effets possibles d'une «fièvre schmittienne» (shimite re) restée peu comprise. Mais c'est le thème du «classique» (gudian) qui reste l'aspect le plus visible de l'enseignement de ce professeur: ainsi, à l'Université Sun Yat-sen de Canton fonctionnent, sous sa direction, de véritables ateliers de traduction donnant lieu à des versions chinoises commentées de classiques grecs et latins... Le but d'une telle entreprise n'est pas seulement de faire œuvre d'érudition en redécouvrant les textes fondamentaux de la tradition occidentale, il est d'encourager la renaissance d'un «ethos classique» se nourrissant du contenu de vérité transmis par les textes des Anciens.

Ce thème du classique (gudian) puisé dans l'Antiquité européenne peut-il aussi trouver son application dans le domaine chinois, avec la littérature canonique (jingdian) qui lui est spécifique? Liu Xiaofeng le pense, car le retour à la vérité classique peut également s'exercer au sein de la tradition chinoise. S'il critique la grande entreprise philosophique du «néo-confucianisme contemporain», c'est parce que celle-ci reconstruit la pensée confucéenne selon les

16. «Liberal education is the necessary endeavour to found an aristocracy within democratic mass society», Strauss Leo, «What is liberal education?», in Liberalism Ancient and Modern, University of Chicago Press, 1968, p. 5 (on notera que le double discours straussien se retrouve dans le titre même de cet essai, puisque «libéral» ne renvoie pas au libéralisme moderne, mais en réalité à l'homme libre de la cité antique). 
normes de la philosophie occidentale moderne, inspirée des Lumières. Il faut au contraire s'efforcer de retrouver les vérités enfouies dans les textes anciens, par delà les exégèses innombrables de la tradition lettrée. Dans cette perspective, la tradition du Gongyang fournit des enseignements précieux sur le sens ultime des classiques chinois. Dans une discussion serrée avec le sinologue allemand Wolfgang Kubin, Liu Xiaofeng s'efforce ainsi de montrer que le paradigme révolutionnaire n'est pas une importation moderne occidentale mais se trouve déjà à l'œuvre au sein de cette tradition ${ }^{17}$. On voit que des rapprochements sont ainsi possibles entre la posture anti-moderne de Liu et le confucianisme inspiré du Gongyang défendu par Jiang Qing: le premier a, de fait, encouragé le second à intégrer à cette tradition particulière des réflexions de la théologie politique occidentale ${ }^{18}$.

On voit ainsi la complexité de l'attitude «classiciste» défendue par cet auteur et la richesse des horizons de pensée ainsi mobilisés, tant intellectuels que politiques, au-delà de ce qui ne pourrait apparaître que comme une simple défense des humanités dans l'université moderne.

Si l'on choisit de finir cette évocation des modifications survenues dans les années 2000 dans la perception de l'historicité en présentant certaines propositions mises en avant par Gan Yang, c'est moins en raison de leur intérêt théorique que de leur valeur de symptôme. Cet auteur, en effet, est avant tout aujourd'hui préoccupé par une vision continuiste de l'histoire, dans le but de proposer un tableau de la Chine actuelle pouvant tirer profit de trois légitimités successives. Son raisonnement procède en deux temps. Ce qu'il faut d'abord établir, au rebours des conceptions communément reçues, c'est une continuité logique entre les trente premières années et les trente dernières années de la république populaire. Loin de signifier une rupture absolue, l'ère des réformes ouverte par Deng Xiaoping n'aurait été possible que par les conséquences de la «création destructrice» de l'époque maoïste: celle-ci, en effet, par son démantèlement de toute économie centralisée et planifiée, aurait eu pour effet non intentionnel de

17. Cf. Rujia yu minzu guojia (Confucianisme et État-Nation), Beijing, Huaxia chubanshe, 2007.

18. $C f$. Liu Xiaofeng, «Hanyu shenxue yu lishizhexue» (Théologie en langue chinoise et philosophie de l'histoire), in Shengling jianglin de xushi (L'Avènement de l'esprit saint), Beijing, Sanlian shudian, 2003 : certains courants de la pensée chinoise aujourd'hui pourraient bénéficier des apports de la théologie politique occidentale, en particulier «le confucianisme politique inspiré par la tradition du Gongyang» (p. 55-56). Et la préface à Liu Xiaofeng (éd.), Dangdai zhengzhishenxue wenxuan (A Reading of Contemporary Political Theology), Jilin renmin chubanshe, 2002, où Liu explique les conditions dans lesquelles il a confié à Jiang Qing la traduction en chinois d'essais occidentaux sur la théologie politique. 
rendre possible, en contraste avec la stagnation de la Russie post-soviétique, le dynamisme du nouveau capitalisme chinois... Mais ces deux périodes, à leur tour, ne font sens que si l'on prend en compte un héritage beaucoup plus vaste, celui de l'époque impériale, ramené pour l'essentiel au confucianisme. De là la mise en place d'un montage très particulier, celui de «trois traditions » : la tradition de la réforme (ayant pour cœur l'idée de marché et l'encadrement juridique qui lui correspond), la tradition maoïste (mettant l'accent sur l'égalité et la justice) et la tradition confucéenne, soucieuse notamment, comme on l'a vu, du rôle joué par les relations interpersonnelles. C'est la conjonction acceptée de ces trois «traditions » qui formerait désormais le «nouveau consensus » de la future République socialiste confucéenne.

Pour enraciner encore davantage cette construction idéologique dans la tradition culturelle, Gan Yang n'hésite pas à en appeler, lui aussi, à une tradition exégétique dont on a déjà pu constater le prestige aujourd'hui renouvelé, celle du Gongyang, et à sa conception d'une «continuité de trois traditions ou légitimités» (tong san tong) ${ }^{19}$. Il écrit ainsi, dans l'article présenté ici: «Ce qu'il faut souligner, c'est que les traditions de Confucius, de Mao Zedong et de Deng Xiaoping renvoient à une continuité, celle de la civilisation et de l'histoire chinoise. Dès lors, nous devons réexaminer les succès de la réforme en l'inscrivant dans une relation de continuité avec l'ère maoïste, et nous devons aussi prendre la mesure du caractère fondateur de la civilisation et de l'histoire traditionnelles chinoises pour la Chine moderne». On notera, s'agissant d'un texte polémique plus que d'une analyse scientifique, que si cette perception stratifiée de l'historicité a pour mérite de faire entrer de nouveau l'expérience du totalitarisme maoïste dans la nouvelle mémoire politique, une telle opération ne peut se faire qu'au prix de simplifications ou d'amnésies nouvelles : la complexité de l'expérience impériale et la créativité tant politique qu'institutionnelle

19. Gan Yang, Tong san tong (Unir les trois traditions), Beijing, Sanlian, 2007. Il peut être intéressant de noter la manière dont l'un des protagonistes de notre débat, le partisan de la «Nouvelle gauche», Wang Hui, définit lui-même le sens de cette notion: «Les san tong signifient les orthodoxies des trois dynasties antiques des Xia, des Shang et des Zhou, gouvernées par des rois-sages. L'enseignement de l'école du Gongyang soutient que ces trois orthodoxies s'identifiaient les unes aux autres. Ainsi, le sens littéral de l'expression tong san tong est celui de la liaison de ces trois orthodoxies. Néanmoins, cette conception implique qu'une maison royale particulière ne peut maintenir sa domination pour toujours et justifie ainsi le remplacement incessant d'une dynastie par une autre. Elle appelle également chaque famille régnante à faire preuve de bienveillance envers la précédente, puisqu'elle sera un jour elle-même remplacée»... Wang Hui, «The Liberation of the Object and the Interrogation of Modernity, Rethinking the Rise of Modern Chinese Thought», Modern China, vol. 34, 1, janvier 2008, p. 140. 
La Chine des années 2000: regards nouveaux sur le politique

de l'époque républicaine (entre les deux guerres mondiales) passent ainsi à leur tour au second plan...

\section{Entre nation et monde}

Une question essentielle de ce débat mérite sans doute d'être explicitée: au-delà de la variété de leurs perspectives, à quelle communauté réelle ou imaginaire ces auteurs se réfèrent-ils ? Quel est l'horizon dans lequel se déploie leur réflexion politique? Ces réexamens de la question du politique concernent-ils essentiellement une nation ou une culture, ou bien prétendent-ils à une validité plus générale, voire universelle?

Au risque d'un certain schématisme, on distinguera les auteurs qui privilégient une perspective exclusivement chinoise et ceux chez qui un horizon d'universalisation se trouve davantage affirmé, pour des raisons qui peuvent être diverses. Et l'on choisira de s'arrêter davantage, pour finir, sur le cas de Wang Hui, en raison de l'originalité de sa position.

Que la spécificité d'une «voie chinoise» soit mise en avant ne surprendra pas dans le cas du confucianisme politique d'un Jiang Qing. En effet, un des fondements de la légitimité de l'ordre politique qu'il imagine se trouve dans l'exigence d'une continuité historique et culturelle, contre ce qui constitue selon lui l'artificialisme de constructions rationnelles comme les systèmes exclusivement démocratiques. Certes, l'ancienne tradition confucéenne dont il se réclame comportait bien un horizon universaliste: l'idéal civilisateur et ritualiste ne concernait pas qu'un peuple ou une nation, mais l'univers tout entier. Cependant, le contexte politique dans lequel s'exerce la réflexion contemporaine incite à un plus grand réalisme et les partisans les plus inconditionnels du confucianisme ne peuvent que reconnaître la pluralité des civilisations et des cultures: envers celles-ci, on se borne à exprimer le souhait vague d'une coexistence pacifique et d'une influence mutuellement enrichissante.

La dimension nationaliste du nouveau confucianisme se fait en revanche plus explicite chez un auteur comme Kang Xiaoguang. Bien qu'il n'ait pas été possible d'inclure un article de ce dernier dans le présent recueil, il peut être utile de donner une idée de cette position radicale. Né en 1963, Kang Xiaoguang est un professeur de l'Université du Peuple, de formation scientifique, qui n'a découvert que sur le tard le confucianisme. Lecteur de Huntington, c'est dans le cadre d'une doctrine systématique sur la nécessité d'un «nationalisme culturel» (wenhua minzuzhuyi) qu'il milite en faveur d'un confucianisme qui serait reconnu par les autorités comme religion ou enseignement national (guojiao). Cette religion ainsi reconstruite contribuerait à assurer la cohésion nationale et à faire obstacle aux doctrines étrangères (à la menace chrétienne, par exemple). 
Chez cet auteur d'un livre sur La Voie chinoise, c'est l'idéologie nationaliste qui met à son service une version instrumentalisée du confucianisme. La dimension potentiellement universaliste de ce dernier passe ici complètement à l'arrière-plan ${ }^{20}$.

On a vu le caractère parfois insaisissable de l'attitude d'un Gan Yang, en raison notamment de la diversité de ses prises de position successives. En dépit de son ouverture intellectuelle, la logique de sa conception nouvelle d'une «république socialiste confucéenne» le conduit lui aussi, dans le texte proposé dans ce recueil, à mettre en valeur les «conditions spécifiques du pays » (guoqing) et défendre, en définitive, la nécessité d'une «voie chinoise» (Zhongguo daolu). Chez cet ancien «occidentaliste», on peut lire dorénavant: «Je ne pense pas que toutes ces prétendues théories occidentales puissent avoir grande valeur pour nous; les Chinois doivent utiliser leur tête pour penser et leurs pieds pour avancer...»

Il est vrai que le désir légitime de trouver dans la tradition chinoise des ressources intellectuelles pour penser les problèmes non seulement de la Chine, mais aussi du monde, se heurte à des difficultés considérables en raison d'une universalisation de fait des conceptions politiques occidentales au cours du siècle dernier. Cette difficulté pourrait être illustrée par une tentative récente, non représentée dans ce recueil mais qui a provoqué un large débat, celle du philosophe Zhao Tingyang, désireux de promouvoir dans les relations internationales l'antique concept du Tianxia, c'est-à-dire de l'univers ou, plus précisément, du «tout-sous-le-Ciel». Cette notion, apparue sous la dynastie archaïque des Shang, pouvait dénoter, selon le cas, l'espace où s'exerçait l'autorité d'une royauté universelle ou l'ensemble de l'univers alors connu.

Le point de départ de Zhao est en effet le constat que notre monde reste un non-monde. C'est un monde failli (failed world) en l'absence d'une pensée et d'une institution permettant d'appréhender le monde comme monde et non comme un assemblage de communautés politiques particulières : «Nous manquons d'une pensée du monde (worldism) en tant que monde; celle-ci ne peut être ni l'universalisme (qui n'est que l'expression du nationalisme des États les plus avancés) ni le pluralisme (comme nationalisme de résistance des nations les plus faibles). » Or, la reprise critique de l'antique concept du Tianxia,

20. Cf. Sébastien Billioud et Joël Thoraval, «Anshen liming ou la dimension religieuse du confucianisme», Perspectives chinoises, $\mathrm{n}^{\circ}$ 2008/3, p. 96-116; David Ownby, «Kang Xiaoguang and the promotion of China's Confucian Revival», China Perspectives, $n^{\circ}$ 2009/4. Parmi les ouvrages écrits par Kang Xiaoguang, voir Ren zheng, Zhongguo zhengzhi fazhan de di santiao daolu (Gouverner par le sens de l'humain. La troisième voie du développement politique chinois), Singapour, Global Publishing, 2005 ; Zhongguo de daolu (La voie chinoise), ouvrage non officiellement publié, 2003. 
du «tout-sous-le Ciel», permet d'offrir l'idéal d'un ordre à la fois holistique, inclusif et harmonieux supérieur à la perspective occidentale dont l'aspiration la plus haute reste la conception agonistique d'un espace inter-national où les États-nations continuent de facto de jouer un rôle décisif. Selon cet auteur, une réinterprétation moderne de cette notion permettrait d'ajouter la dimension de la mondialité à la simple internationalité: elle articule, en effet, à la notion spatiale d'univers l'idée d'une reconnaissance de tous les peuples et celle d'une institution rendue ainsi pleinement légitime. «Le concept de tout-sous-le-Ciel, écrit-il, est une vision du monde dans laquelle le «monde» est compris comme étant l'unité d'un monde physique (la terre), d'un monde psychologique (le sentiment général des peuples) et d'un monde institutionnel (une institution mondiale) ${ }^{21}$. Dans un tel univers inclusif, fondé sur une éthique universelle, il n'existerait plus d'Autre irréductible (personne n'aurait plus le statut politique d' «étranger» ou le statut religieux de «païen») et la relation schmittienne et absolue de l'ami/ennemi serait remplacée par la notion chinoise et relative d'éloignement et de proximité... En un mot, l'adoption de la notion chinoise de Tianxia par la communauté internationale permettrait de compléter la «grammaire politique» moderne des relations internationales, accusée d'être restée trop élémentaire parce que gouvernée par l'antagonisme des États-nations et de leurs associations.

Indépendamment des difficultés intrinsèques de cette philosophie du monde, on ne peut que noter la contradiction performative d'une telle entreprise qui, au nom du dépassement de la nation et d'une responsabilité envers «le monde comme monde», n'en promeut pas moins une vision proprement chinoise: celleci trouve au moins partiellement son origine dans la conscience renouvelée d'un nationalisme culturel encouragé par l'actuelle mondialisation ${ }^{22}$.

Si des auteurs sélectionnés dans ce recueil axent leur réflexion politique avant tout sur l'expérience nationale et privilégient la définition d'une voie proprement

21. Zhao Tingyang, Tianxia tixi (Le système du Tianxia), Jiangsu jiaoyu chubanshe, Nanjing, 2005 et «La philosophie du Tianxia», Diogène, n² 221, janvier 2008, p. 4-25.

22. Sur le contexte politique de cette entreprise, voir Ji Zhe, «Tianxia, retour en force d'un concept oublié», 2008, La Vie des idées, (http://www.laviedesidees.fr/Tianxia-retouren-force-d-un.html). Quant à la difficulté théorique principale d'une telle opération intellectuelle, elle est sans doute de prétendre proposer comme concept opératoire, dans le cadre sécularisé et moderne des relations internationales, une antique notion de nature indissociablement politique et religieuse : la relation dynamique du Ciel avec la terre est impensable sans la médiation d'un personnage essentiel, celui de l'Empereur, monarque universel seul habilité à rendre un culte au Ciel, à l'autel qui lui est destiné. Faute d'une réflexion rigoureuse sur le processus de sécularisation affectant ces notions impériales, la question de leur éventuelle légitimité moderne reste du domaine de l'imaginaire. 
chinoise, d'autres au contraire insèrent clairement leur travail dans un horizon ouvert sur l'universel. C'est le cas de Lee Ming-huei, dont le confucianisme libéral présuppose l'existence d'un environnement démocratique et pluraliste. La perspective confucéenne est compatible avec la vision d'un John Rawls d'une «société bien ordonnée »: elle n'est qu'une de ces reasonable comprehensive doctrines capables de pleinement développer leurs potentialités tout en se prêtant, lorsqu'elles sont confrontées à des conceptions différentes ou rivales, à des «consensus par recoupement» (overlapping consensus), au nom d'un idéal de justice comme équité. Le fait que les écrits de Lee Ming-huei ne relèvent pas seulement de la spéculation philosophique mais soient également le produit d'une expérience vécue, celle du processus de démocratisation de la société taiwanaise, ne donne que plus de poids à ses prises de position. Cet horizon universaliste est aussi présent dans la pensée de Qin Hui, qui perçoit dans l'expérience chinoise d'aujourd'hui des problèmes parfaitement similaires à ceux que connaissent d'autres régions du monde, qu'il s'agisse de liberté politique ou de justice sociale. Sa connaissance de l'univers chinois (mais aussi russe) lui permet de présenter en des termes parfaitement contextualisés des conceptions politiques où l'on peut reconnaître une inspiration social-démocrate.

Il est un universel d'une autre sorte et plus paradoxal, qu'illustre ici Liu Xiaofeng, et auquel il parvient, en quelque sorte, par une voie négative. La vision méta-historique qui est la sienne le préserve en principe de tout relativisme nationaliste ou culturaliste. Il s'exclame ainsi, de manière provocatrice, en conclusion d'un texte sur la signification absolue de l'événement christique, et sur l'incommensurabilité de cet événement avec la continuité de la tradition culturelle chinoise: «Je suis un profanateur du Tao!» (dao zhi fei $)^{23}$. En tout état de cause, à supposer que la singularité absolue de cet événement ouvre la possibilité d'un universel, ce dernier est malgré tout d'une nature particulière car il est fondamentalement pré-politique. Il est moins l'objet d'une philosophie que d'une théologie politique. Quant au retour proclamé, dans un esprit straussien, aux vérités éternelles des Anciens, il ouvre également un espace supra-historique qui, certes, n'est pas sans soulever de nombreux problèmes, tant philosophiques que pratiques, mais qui a au moins pour conséquence nécessaire de ne reconnaître aucune suprématie de principe à l'Occident ou à la Chine.

Mais il est une autre possibilité de penser le rapport entre le national, le régional et le global, que la réflexion de Wang Hui permet de mettre en place.

23. Liu Xiaofeng, «Hanyu shenxue yu lishizhexue» (Théologie en chinois et philosophie de l'histoire), in Shengling jianglin de xushi (Avènement de l'esprit saint), Sanlian shudian, 2003, p. 107. 
Né en 1959 à Yangzhou, Wang Hui poursuit des études littéraires à Pékin, sur le grand écrivain Lu Xun, avant de se trouver mêlé de manière active, en 1989, aux manifestations de la place Tian'anmen. Dans le contexte de la répression brutale de ce mouvement, il est envoyé plusieurs mois en rééducation dans la province du Shanxi. Cette expérience est pour lui une révélation: il découvre la misère des campagnes, la gravité des inégalités sociales et les effets pervers du démantèlement hâtif du système de protection sociale de la période maoïste. Cette prise de conscience contribuera à nourrir son aspiration à plus de justice sociale. Revenu à Pékin en 1991, il est le co-fondateur (avec Wang Shouchang et Chen Pingyuan) du périodique Xueren (Scholar), qui se caractérise à la fois par l'ouverture intellectuelle et le sérieux académique. Parallèlement, il entreprend une longue relecture de l'histoire intellectuelle de la Chine moderne, qui donnera lieu aux quatre gros volumes de L'Essor de la pensée chinoise moderne ${ }^{24}$. Cette ambitieuse entreprise se donne notamment pour objectif de remettre en cause les catégories d'origine occidentale, devenues dogmatiques dans les discours officiels relatifs à la «modernisation» de la Chine, et se situe dans la continuité d'un projet intellectuel visant à «dépasser les divisions binaires auxquelles les intellectuels chinois se sont habitués depuis longtemps déjà (Chine/Occident ; tradition/modernité), [...], procéder à un nouvel examen critique des conditions historiques de la quête chinoise de la modernité et de ses modalités et penser la question chinoise en la replaçant dans une perspective historique de mondialisation ${ }^{25}$.

Mais ce sont ses fonctions de rédacteur en chef du mensuel Dushu (Lire), de 1996 à 2007, qui vont permettre à Wang Hui de jouer un rôle d'intellectuel public et de développer ainsi les thèmes qui lui vaudront l'appellation de porte-parole d'une «nouvelle gauche». Bien qu'étant à l'origine une étiquette péjorative inventée par le camp libéral pour suggérer l'existence d'une complicité de fait avec l'ancienne gauche, c'est-à-dire le Parti communiste, cette appellation a fini par être acceptée par un courant d'intellectuels mettant en question l'attention exclusive apportée par les libéraux à la sphère des droits et des libertés. Wang exprime la différence en toute clarté: "Les libéraux soutiennent le règne du marché dans l'économie comme la seule voie correcte pour la Chine. À leurs yeux, c'est seulement l'absence de réforme politique qui pervertit le bon fonctionnement du marché, mais si la constitution était révisée pour protéger les

24. Wang Hui, Xiandai zhongguo sixiang de xingqi (L'essor de la pensée chinoise moderne), Beijing, Sanlian shudian, 2004.

25. Voir Wang Hui, Dangdai Zhongguo de sixiang zhuangkuang yu xiandaixing wenti (L'état de la pensée en Chine contemporaine et la question de la modernité), http:// www.zhuoda.org/luotuo8895/43408.html, consulté le 27 juin 2009. Ce texte avait été publié à l'origine dans le numéro 1997/5 de la revue Tianya. 
droits des citoyens, nous aurions alors une société raisonnablement égalitaire et un degré satisfaisant de justice sociale. À mon avis, c'est une illusion. La démocratie politique ne sera pas produite par un marché devenu légalement impartial, mais par la force de mouvements sociaux contre l'ordre existant. Ce point est central dans la généalogie du travail intellectuel critique qui est à présent identifié sous le nom de Nouvelle gauche ${ }^{26}$.»

La question du rôle des médias que Wang Hui aborde dans son article n'est qu'une illustration de ce qu'il nomme un processus de «dépolitisation». Bien qu'il soit redevable de ce concept à Carl Schmitt, qui désignait sous ce terme la tendance inévitable du libéralisme à dissimuler l'essence du politique (comme décision relative à la relation ami/ennemi) sous l'apparence faussement neutre de l'économie ou de la morale, Wang en fait un usage critique dans une perspective que l'on peut qualifier de néo-marxiste ${ }^{27}$. En dépit de la différence des contextes culturels et historiques, il croit pouvoir déceler tant en Occident qu'en Chine un processus comparable de dépolitisation qui transforme les partis politiques, autrefois vecteurs d'intérêts et de valeurs antagonistes, en simples rouages d'un État devenu technocratique et fonctionnalisé. Sous la fausse objectivité du développement économique ou de la bonne gouvernance, l'État dissimule les choix qu'il opère en faveur des intérêts de telle ou telle catégorie sociale et interdit aux citoyens la possibilité de faire entendre des intérêts divergents dans un débat ouvert et contradictoire. En effet, souligne Wang, bien que la critique post-moderne ait mis à juste titre l'accent sur le caractère «construit» de la notion de classe sociale, les antagonismes sociaux analysés autrefois par Marx n'en reste pas moins une réalité.

La question de l'usage des médias en Chine, abordée dans ce recueil, est ainsi un exemple du débat qui oppose la nouvelle gauche au camp libéral. Pour la première, il n'est pas suffisant de réclamer davantage de transparence et de liberté de l'espace public. Il faut prendre en compte le fait que ce dernier est en permanence un champ de forces procédant par exclusion et hiérarchisation. Il faut donc se demander: «Reconnaît-on ou non des relations de pouvoir derrière l'espace public? Est-on prêt à dévoiler le jeu des intérêts derrière les revendications de "publicité" (gonggongxing), c'est-à-dire de dimension publique des débats ?... » Dans une référence à Gramsci et au courant post-colonial des «subaltern studies» indiennes, Wang Hui pose notamment la question de la participation effective des catégories exclues, que la «posture de pure critique

26. Wang Hui, «The new criticism», in Wang Chaohua, One China, Many Paths, Londres, Verso, p. 64

27. Voir Wang Hui, Qu zhengzhihua de zhengzhi (Une politique dépolitisée), Beijing, Sanlian shudian, 2008 (notamment, sur le rapport à Schmitt, p. 43-44). 
morale des médias », qui caractérise une certaine élite intellectuelle, ne suffirait pas à résoudre: «Donner la parole à ceux qui sont sans voix est, à l'échelle du monde, le plus grand défi auquel les médias ont à faire face.»

On peut relever au moins trois conséquences d'une telle attitude. La première concerne le rôle qui se trouve dévolu aux intellectuels critiques. À l'exemple des luttes menées actuellement en Chine pour la défense des droits de catégories spoliées (weiquan yundong), les intellectuels doivent quitter leur tour d'ivoire et «prendre appui sur l'interaction entre débat public, mouvements sociaux et innovation institutionnelle», dans un mouvement «d'aller et retour entre théorie et pratique dans leurs recherches». Une seconde conséquence est que, en réaction contre la dépolitisation du pouvoir communiste actuel dont la fausse neutralité dissimule le risque d'un «nouveau despotisme social», l'époque maö̈ste fait l'objet d'une réhabilitation relative. Tout en étant critique sur les violences arbitraires dont il a pu faire lui-même au moins indirectement l'expérience, Wang Hui croit pouvoir réévaluer de manière positive les phénomènes de "grands débats » et de «mouvements de masse » qui permettaient d'articuler explicitement les antagonismes politiques. Il s'élève contre la censure dont font l'objet les recherches sur la Révolution culturelle, devenue selon lui une «zone interdite ${ }^{28}$. Sans méconnaître la question des droits mise en avant par les libéraux, Wang privilégie une conception agonistique du politique, comme «processus par lequel différents groupes d'intérêts confrontent leurs appréciations en se fondant sur des valeurs différentes, processus nécessairement différencié, visant à échapper au contrôle et en extension continuelle»...

Une troisième conséquence concerne le thème plus général évoqué ci-dessus : quel espace, quel horizon pour la pensée du politique ? Une des caractéristiques constantes de l'attitude de Wang Hui est la mise en question du cadre de l'Étatnation. Il y a deux justifications à cette position. La première est le produit de sa réflexion sur l'histoire intellectuelle de la Chine moderne que, dès son étude sur Lu Xun, il a souhaité situer dans l'espace plus large du contexte asiatique. De fait, Wang Hui est l'un des rares intellectuels critiques chinois entretenant un dialogue suivi avec le milieu académique japonais. Il est notamment le partenaire d'une discussion collective sur «l'Asie» comme espace imaginaire et comme notion idéologique ${ }^{29}$. La seconde justification est plus importante et tient au diagnostic porté très tôt sur le "problèmes chinois » : la raison dernière des problèmes sociaux dont souffre aujourd'hui la Chine n'est à trouver ni dans

28. Wang Hui est en relation avec la gauche radicale occidentale, et s'intéresse notamment à l'« International Center for the study of the Cultural Revolution» lancé par le philosophe Alain Badiou et le sociologue italien Alessandro Russo.

29. Cf. en français Wang Hui, «Les Asiatiques réinventent l'Asie», in Le Monde diplomatique, février 2005. 
la tradition culturelle ni dans l'idéologie du régime actuel, mais avant tout dans les effets d'une insertion brutale dans le système d'un capitalisme mondialisé ${ }^{30}$. Dès lors, la réflexion sur le politique ne peut plus être limitée à l'espace national : elle n'acquiert de sens que déployée à l'échelle mondiale.

Dans l'ensemble des auteurs ici représentés, Wang Hui défend donc une voie qui n'est ni celle d'un nationalisme culturel, ni celle d'un universalisme libéral, mais celle d'un alter-mondialisme radical.

\section{Conclusion}

À l'issue de ce bref parcours de la réflexion politique en Chine aujourd'hui, on se limitera à quatre remarques.

Il faut en premier lieu souligner combien le tableau ici présenté n'est que partiel. Attentif à ce que la décennie 2000 présentait de nouveau par rapport aux débats intellectuels antérieurs, ce recueil consacre une place plus limitée aux représentants de la pensée libérale et démocratique. Celle-ci, il est vrai, est aujourd'hui mieux connue en raison de nombreuses études de qualité auxquelles il est possible de se reporter ${ }^{31}$. On rappellera néanmoins l'appartenance foncière

30. C'est là que réside la divergence fondamentale entre la nouvelle gauche et les intellectuels libéraux perpétuant, dans les années 2000, l'idéal des «nouvelles Lumières chinoises » des années 1980. Wang Hui écrit ainsi : «En cette époque de capitalisme transnational, la critique des «nouvelles Lumières» s'est exercée à l'égard de facteurs politiques et sociaux (en particulier l'action des États) appréhendés dans une perspective limitée, celle de l'État-nation. Les «nouvelles Lumières » n'ont pas su à temps transformer leur critique de la dictature de l'État en une analyse des relations complexes État - société au cours du processus de constitution d'un marché capitaliste. De surcroît, elles ne sont pas à même d'analyser en profondeur les changements dans le pays qui sont liés à ce contexte de marché. Ce mouvement n'a pas véritablement compris que la question chinoise est désormais aussi celle du capitalisme mondial. De ce fait, son diagnostic sur les problèmes chinois devrait être en même temps un diagnostic sur le capitalisme qui se mondialise jour après jour, et sur ses problèmes. Le mouvement ne peut plus, comme dans le passé, se référer simplement à l'Occident comme unique étalon dans la critique de la culture et de la politique propres à la société chinoise». Wang Hui, Dangdai Zhongguo de sixiang zhuangkuang yu xiandaixing wenti, op. cit.

31. Voir par exemple l'article récent de Feng Chongyi, «Les libéraux chinois dans la Chine post-Tiananmen», Perspectives chinoises n² 2009/2, p. 32-45; l'étude de Ren Jiantao, Zhongguo xiandai sixiang mailuo zhong de ziyouzhuyi (Le libéralisme dans les lignées de la pensée contemporaine en Chine), Pékin, Beijing daxue chubanshe, 2004 ; l'ouvrage de Jean Philippe Béja, À la recherche d'une ombre chinoise, le mouvement pour la démocratie en Chine (1919-2004), Paris, Le Seuil, 2004. Pour une présentation des vues des intellectuels libéraux par eux-mêmes, voir, parmi d'autres, l'article de Liu Junning, «Classical Liberalism Catches On in China», Journal of Democracy, vol. 11, 
de Qin Hui à ce courant, en dépit, ou en raison, des inflexions social-démocrates de sa réflexion.

En second lieu, il semble possible de retenir, au sein de ce débat complexe et en évolution permanente, au moins trois innovations majeures, caractéristiques de la présente décennie. D'abord, il est remarquable que la question des rapports entre confucianisme et démocratie ait fait retour avec une telle force dans les discussions intellectuelles de la Chine continentale. Si cette discussion peut emprunter certains arguments à des débats plus anciens, la référence aux valeurs confucéennes se situe désormais au-delà des abstractions culturalistes pour aborder des questions pratiques et institutionnelles. Ensuite, le rapport de la pensée politique au passé, proche ou lointain, s'est également modifié. Dans un univers intellectuel souvent partagé entre l'utopisme et le pragmatisme (sous le contrôle resté vigilant de l'idéologie), des perceptions nouvelles de l'historicité se font jour, qu'il s'agisse du retour critique sur le thème du «moderne» ou d'une vision renouvelée du passé maoïste, notamment au sein de la nouvelle gauche. Enfin, en dépit de la persistance d'un face-à-face intellectuel entre «Chine et Occident» (ce dernier étant trop souvent ramené à la seule Amérique), on assiste dans l'imaginaire collectif à une sorte de décentrement progressif de la référence occidentale. Ce peut être le produit d'une confiance renouvelée dans la spécificité d'une "voie chinoise» se réclamant de manière diverse d'une «tradition» réinterprétée, de la recherche d'un universalisme véritable capable de faire droit aux différences de la culture autochtone (comme chez Lee Ming-huei) ou encore de la participation active à des échanges "globalisés» ayant pour effet de «provincialiser l'Europe» (D. Chakrabarty) au nom d'un alter-mondialisme radical.

Pour autant, la différence des points de vue exprimés ici laisse néanmoins percevoir l'existence de rapprochements, voire de complicités parfois paradoxales, qui tiennent autant à des relations d'amitié ou de sensibilité générationnelle (Gan Yang, Liu Xiaofeng, Jiang Qing...) qu'à l'utilisation de ressources intellectuelles communes à des fins qui peuvent être parfois contradictoires. On a ainsi pu noter combien l'appel fait à des traditions anciennes comme celle du Gongyang pouvait être le fait d'intellectuels se réclamant du confucianisme politique, d'une pensée anti-moderne ou d'une nouvelle gauche. On pourrait également évoquer l'usage contrasté des références faites à Schmitt, ainsi mis au service, d'une manière qui n'est pas propre qu'à la Chine, de pensées ultraconservatrices ou inspirées au contraire par un progressisme radical. S'ajoutant à l'opacité relative produite par un environnement idéologique exerçant toujours

$\mathrm{n}^{\circ} 3$, juillet 2000, et celui de Xu Youyu, «Libéralisme vs. Nouvelle gauche: état des lieux », La vie des idées, $\mathrm{n}^{\circ} 17,2006$, p. 77-88. 
sa censure, la circulation de ces références et de ces thématiques ne peut que contribuer à la complexité du débat en cours, dont il convient à chaque fois de restituer tant la généalogie que la particularité contextuelle.

Il faudrait s'interroger pour finir sur les limites de ces discours intellectuels ou, plus exactement, sur les relations qu'ils peuvent entretenir avec les mouvements plus profonds, de nature collective, qui travaillent la société. Si l'on met à part le cas de Taiwan, où le débat politique peut aujourd'hui se développer dans le cadre d'un espace public relativement transparent, on pourrait distinguer, sur le continent, plusieurs situations possibles. À côté de l'élitisme revendiqué d'un Liu Xiaofeng, se manifeste parfois la volonté d'une articulation de la réflexion intellectuelle et des mouvements sociaux, qu'il s'agisse d'entreprises se réclamant de la tradition, dans le domaine de l'éducation notamment (Jiang Qing, dans une certaine mesure), ou de la défense démocratique des droits de populations défavorisées (Qin Hui ou Wang Hui, chacun à sa manière). Mais on ne peut ignorer non plus la relation de négociation que peuvent (ou que doivent) entretenir ces différents protagonistes avec le pouvoir: en particulier, ces dernières années ont vu s'opérer un rapprochement de fait entre une partie de la nouvelle gauche et les entreprises de l'«ancienne gauche» communiste, dont le slogan de Gan Yang sur une république socialiste confucéenne, se fait à sa manière l'écho. 


\section{GLOSSAIRE}

Bense shenxue 本色神學

Chen Pingyuan 陳平原

Dao zhi fei 道之罒

Dong Zhongshu 董仲舒

Dushu 讀書

Gan Yang 甘陽

Gonggongxing 公共性

Gongyang 公羊

gudian 古典

guojiao 國教

guoqing 國情

guoxue re 國學熱

hanyu shenxue 漢語神學

Jiang Qing 蔣慶

jingdian 經典

Kang Youwei 康有為

Lee Ming-huei 李明輝

Liu Xiaofeng 劉小楓

Lu Xun 魯迅

Mou Zongsan 牟宗三

neisheng 內聖

Qin Hui 秦暉

Shimite re 施密特熱

Tang Junyi 唐君毅

tianxia 天下

Tong san tong 通三統

Wenhua: Zhongguo yu shijie 文化: 中國與世界

waiwang 外王

Wang Hui 汪暉

weiquan yundong 維權運動

wenhua jidujiao 文化基督教

wenhua minzuzhuyi 文化民族主義

xin zuopai 新左派

Xu Fuguan 徐復觀

xueshu 學術

Xueren 學人

Zhang Junmai 張君勱

Zhang Xianglong 張祥龍

Zhao Tingyang 趙汀陽

zhengdao 政道

zhidao 治道

zhongguo daolu 中國道路

ziwo kanxian 自我坎陷 